\title{
Formulários de notificação de reações adversas a medicamento nos sistemas de farmacovigilância do Brasil e outros doze países latino-americanos: análise comparativa
}

\author{
Adverse drug reaction notification forms of the pharmacovigilance \\ systems in Brazil and twelve other Latin-American countries: \\ a comparative analysis
}

Daniel Marques Mota (http://orcid.org/0000-0003-4156-0138) ${ }^{1}$

Álvaro Vigo (http://orcid.org/0000-0002-5797-9470) ${ }^{2}$

Ricardo de Souza Kuchenbecker (http://orcid.org/0000-0002-4707-3683) ${ }^{2}$

${ }^{1}$ Agência Nacional de Vigilância Sanitária. SIA Trecho 05, Guará. 71205050 Brasília DF Brasil. dmarques2003@

yahoo.com.br

${ }^{2}$ Programa de Pós-

Graduação em

Epidemiologia,

Universidade Federal do Rio

Grande do Sul. Porto Alegre

RS Brasil.

\begin{abstract}
The scope of this study was to assess the forms used to report suspected adverse drug reactions (ADR) to the pharmacovigilance system in Brazil and twelve other Latin-American countries. The study comprised three stages. In the first stage, the forms were attributed a score relating to the presence of critical items to generate $A D R$ notification. In the second stage, the variables of the forms were quantified and classified regarding feasibility to contribute to an appropriate assessment of causality. In the last stage, hierarchical clustering was used to identify similar forms. The Venezuelan form achieved the highest score (18 points). The median number of variables in the forms of each country was 41 [26 (Guatemala) to 95 (Brazil)]. Most of the variables of the form were classified as life-critical and the majority contribute as an alternative explanation to causality assessment. Four clusters were identified (1,2, 3 and 4). The forms of Brazil and Bolivia formed two distinct groups, 1 and 3 respectively. The results of this study indicate the need to change the forms of the different countries studied or even delete some variables, making them more appropriate for the process of assessment of ADR causality.

Key words Drug-related side effects and adverse reactions, Pharmacovigilance, Adverse drug reaction reporting systems.
\end{abstract}

Resumo Avaliou-se os formulários de notificação de suspeitas de reações adversas a medicamentos (RAM) do sistema de farmacovigilância do Brasil e outros doze países latino-americanos. O estudo foi composto por três etapas. Na primeira, os formulários foram pontuados em relação à presença de itens essenciais para compor uma notificação de RAM. Na segunda, as variáveis dos formulários foram quantificadas e classificadas, quanto à razoabilidade em contribuírem para a avaliação de causalidade. Na última etapa, recorreu-se à análise de agrupamento hierárquico para identificar os formulários similares. O formulário da Venezuela obteve a maior pontuação (18 pontos). A mediana das variáveis nos formulários dos países foi de 41 [26 (Guatemala) a 95 (Brasil)]. A maioria das variáveis dos formulários foram classificadas como vitais e a maior parte contribui como fator de explicação alternativo para a avaliação de causalidade. Quatro agrupamentos foram identificados (1, 2, 3 e 4). Os formulários do Brasil e da Bolivia formaram dois grupos distintos, 1 e 3 respectivamente. Há necessidade de modificação dos formulários dos países ou até mesmo exclusão de variáveis, tornando-os mais adequados ao processo de avaliação da causalidade das RAM.

Palavras-chave Efeitos colaterais e reações adversas relacionados a medicamentos, Farmacovigilância, Sistemas de notificação de reações adversas a medicamentos 


\section{Introdução}

As reações adversas a medicamentos (RAM) são uma causa frequente, nem sempre previsível, de morbidade e mortalidade ${ }^{1-5}$. Diferentes métodos de monitoramento de RAM na etapa de pós-comercialização têm sido utilizados em diversos estudos $^{6-10}$. A notificação espontânea é, possivelmente, o método mais amplamente utilizado por vários sistemas governamentais de farmacovigilância ${ }^{11,12}$, a exemplo do Sistema Nacional de Farmacovigilância (SINAF) do Brasil ${ }^{13}$.

No âmbito do SINAF, o Sistema Nacional de Notificações para a Vigilância Sanitária, formulário-medicamento que ocasionou danos à saúde (Notivisa-medicamento) é um sistema informatizado online que recebe e armazena as notificações espontâneas de casos suspeitos e confirmados de RAM. O Notivisa-medicamento é gerenciado pela Agência Nacional de Vigilância Sanitária (Anvisa) - órgão nacional de regulação de medicamentos no Brasil - e constitui-se no principal repositório de dados sobre RAM e outros eventos adversos a medicamentos do SINAF.

As RAM notificadas no Notivisa-medicamento passam por um processo de análise realizado por especialistas da Anvisa, objetivando obter informações adicionais para a investigação do caso e avaliação de causalidade nos termos propostos pelo método da Organização Mundial da Saúde $(\mathrm{OMS})^{13,14}$. Este visa atribuir a um medicamento específico, em uma escala de probabilidade qualitativa, a responsabilidade por reações adversas em um paciente ${ }^{13}$. O formulário do Notivisa-medicamento, assim como de outros países, funciona como um filtro na coleta de informações para a qualificação do julgamento causal ${ }^{11,12}$. Consiste, ainda, num componente da farmacogovernança, compreendida como as estruturas de governo, instrumentos de políticas, regramentos, normas e autoridade institucional instituídos e que são gerenciados de maneira a promover os interesses da sociedade quanto à prevenção de eventos adversos relacionados a medicamentos e à segurança dos pacientes ${ }^{15}$.

O objetivo deste estudo foi avaliar comparativamente os formulários do Notivisa-medicamento e de outros doze países latino-americanos (Argentina, Bolívia, Chile, Colômbia, Costa Rica, Cuba, Guatemala, México, Panamá, Peru, Uruguai e Venezuela) que, também, são membros efetivos do Programa Internacional de Monitoramento de Medicamentos da OMS ${ }^{16}$.

\section{Métodos}

Realizou-se um estudo de avaliação em saúde em que as unidades de análise corresponderam aos formulários para a notificação de casos de suspeitas de RAM do Notivisa-medicamento, utilizado por profissionais da saúde, e, também, de outros doze países latino-americanos. A coleta, o processamento e a análise dos dados e das informações compreenderam o período entre novembro de 2015 e março de 2016.

Os dados do formulário eletrônico do Notivisa-medicamento foram obtidos de uma simulação-teste realizada em 27 de novembro de 2015 por um dos autores (DMM), cujo número de notificação gerado automaticamente foi 201511004579. O propósito da simulação foi identificar as informações necessárias à notificação de um caso de uma reação adversa, cuja gravidade resultaria em hospitalização e em óbito do paciente, envolvendo um medicamento suspeito e o uso de um medicamento concomitante. Visto tratar-se de caso hipotético, utilizado à guisa deste estudo, após o término da notificação, esta foi informada à área responsável da Anvisa para a devida exclusão.

Os formulários dos demais países foram identificados nos websites dos órgãos governamentais encarregados das atividades de farmacovigilância da Argentina ${ }^{17}$, Bolívia ${ }^{18}$, Chile $^{19}$, Colômbia ${ }^{20}$, Costa Rica ${ }^{21}, \mathrm{Cuba}^{22}$, Guatemala ${ }^{23}, \mathrm{México}^{24}, \mathrm{~Pa}-$ namá ${ }^{25}$, Peru $^{26}$, Uruguai ${ }^{27}$ e Venezuela ${ }^{28}$. Foram escolhidos os países onde se fala espanhol e que, na época do estudo, figuravam como membros efetivos do Programa Internacional de Monitoramento de Medicamentos da OMS ${ }^{16}$.

Inicialmente, fez-se uma caracterização descritiva dos procedimentos de notificação no Notivisa-medicamento e de seu formulário e da avaliação de causalidade em farmacovigilância conduzida na Anvisa. Nesta direção, foram utilizados documentos referentes aos procedimentos relacionados à notificação e investigação de RAM $^{6,13,29-31}$, que foram recuperados por intermédio de buscas iterativas no website da Anvisa e, também, valeu-se do conhecimento empírico de um dos autores (DMM), que atuou como técnico na área de farmacovigilância dessa instituição no período de 2005 a 2010.

A avaliação comparativa foi estabelecida em três etapas e realizada por um médico (RSK) e um farmacêutico (DMM), com experiências em farmacoepidemiologia e farmacovigilância. Utilizou-se um elenco mínimo de 19 itens considerados essenciais na composição de um formu- 
lário de notificação de suspeita de RAM, o qual foi baseado em estudo que procedeu uma análise comparativa de formulários de outros países ${ }^{11}$ (Quadro 1).

Cada item do formulário recebeu um ponto, perfazendo uma pontuação máxima de 19. Em itens que contivessem dois ou mais aspectos analisados, mas os formulários atendiam apenas parcialmente, a pontuação foi dividida em valores iguais até o valor máximo de 1 ponto (Quadro 1).
Para caracterizar a presença de cada item avaliado, tentou-se ao máximo recuperar informações que atendessem aos quesitos definidos. Por exemplo, para o item "Diagnóstico clínico" foram consideradas variantes, como "Indicação terapêutica" e "Motivo da prescrição/indicação". Em relação ao item "História relevante do paciente", foram pontuados os formulários que mencionavam itens como "Patologia de base" e "Antecedentes clínicos importantes".

Quadro 1. Itens considerados como essenciais em um formulário de notificação de suspeitas de reações adversas a medicamentos (Adaptado de Bandekar et al. ${ }^{11}$ )

\begin{tabular}{|c|c|c|}
\hline Itens essenciais & Informações mínimas requeridas/Definição & Pontos \\
\hline Informações do paciente & $\begin{array}{l}\text { Atributos individuais da pessoa que sofreu a reação adversa: sexo } \\
\text { e idade. }\end{array}$ & 1 \\
\hline Condição de gravidez & Algum item solicitando informação sobre gravidez. & 1 \\
\hline $\begin{array}{l}\text { Conhecimento prévio sobre } \\
\text { alergia }\end{array}$ & Algum item solicitando informação sobre alergia. & 1 \\
\hline Diagnóstico clínico & Alguma menção sobre diagnóstico do paciente. & 1 \\
\hline História relevante do paciente & $\begin{array}{l}\text { Fatos que possam estar relacionados com o problema de saúde } \\
\text { ou o doente, como história médica pregressa, familiar, pessoal e } \\
\text { social. }\end{array}$ & 1 \\
\hline $\begin{array}{l}\text { Informações da reação } \\
\text { adversa }\end{array}$ & $\begin{array}{l}\text { Caracterização da reação adversa, como sinais e sintomas } \\
\text { apresentados pelo paciente e data de início da reação. }\end{array}$ & 1 \\
\hline $\begin{array}{l}\text { Consequência da reação } \\
\text { adversa }\end{array}$ & $\begin{array}{l}\text { Informações sobre o desfecho da reação adversa como óbito, } \\
\text { motivou ou prolongou a hospitalização, anomalia congênita, } \\
\text { efeito clinicamente significativo, etc. }\end{array}$ & 1 \\
\hline Tratamento da reação adversa & Alguma menção sobre tratamento médico da reação adversa. & 1 \\
\hline $\begin{array}{l}\text { Evolução do paciente sobre a } \\
\text { reação adversa }\end{array}$ & $\begin{array}{l}\text { Desfecho final do paciente, como recuperado, cura com sequelas } \\
\text { ou óbito. }\end{array}$ & 1 \\
\hline $\begin{array}{l}\text { Lista de medicamentos } \\
\text { suspeitos }\end{array}$ & $\begin{array}{l}\text { Possibilidade de registrar dados sobre um ou mais medicamentos } \\
\text { suspeitos, excluindo os medicamentos concomitantes. }\end{array}$ & 1 \\
\hline $\begin{array}{l}\text { Dose e frequência do } \\
\text { medicamento(s) suspeito(s) }\end{array}$ & $\begin{array}{l}\text { Forma de uso do(s) medicamento(s) suspeito(s), ou seja, a } \\
\text { quantidade e o número de vezes utilizado a cada dia. }\end{array}$ & 1 ou 0,5 \\
\hline $\begin{array}{l}\text { Data de início e término do } \\
\text { medicamento(s) suspeito(s) }\end{array}$ & Tempo de uso do(s) medicamento(s) suspeito(s). & 1 ou 0,5 \\
\hline $\begin{array}{l}\text { Número do lote e dada de } \\
\text { validade do medicamento(s) } \\
\text { suspeito(s) }\end{array}$ & $\begin{array}{l}\text { Informações sobre o controle de qualidade do(s) medicamento(s) } \\
\text { suspeito(s). }\end{array}$ & 1 ou 0,5 \\
\hline $\begin{array}{l}\text { Lista de medicamentos } \\
\text { concomitantes }\end{array}$ & $\begin{array}{l}\text { Possibilidade de registrar dados sobre um ou mais medicamentos } \\
\text { concomitantes. }\end{array}$ & 1 \\
\hline Ações adotadas & $\begin{array}{l}\text { Conduta adotada em relação ao medicamento suspeito, } \\
\text { como manutenção do uso, ajuste da dose ou substituição do } \\
\text { medicamento. }\end{array}$ & 1 \\
\hline $\begin{array}{l}\text { Suspensão do uso do } \\
\text { medicamento }\end{array}$ & $\begin{array}{l}\text { Houve melhora clínica da reação adversa após a interrupção do } \\
\text { uso do medicamento. }\end{array}$ & 1 \\
\hline $\begin{array}{l}\text { Reintrodução do } \\
\text { medicamento suspeito }\end{array}$ & $\begin{array}{l}\text { A reação adversa reapareceu após o medicamento suspeito ter sido } \\
\text { novamente administrado ao paciente. }\end{array}$ & 1 \\
\hline Causalidade & $\begin{array}{l}\text { Estabelecimento de casualidade do par medicamento-reação } \\
\text { adversa, independentemente do tipo de método utilizado. }\end{array}$ & 1 \\
\hline Dados do notificador & Informações básicas, como nome, telefone e/ou e-mail. & 1 \\
\hline
\end{tabular}


$\mathrm{Na}$ segunda etapa, todas as variáveis de cada formulário, excluindo aquelas relacionadas à identificação do notificador, foram classificadas, quanto à razoabilidade em permitirem adequada avaliação de causalidade em farmacovigilância, compreendendo três categorias ad hoc: i) vital - as variáveis foram consideradas condição sine qua non para a avaliação de causalidade individual; ii) complementar - as variáveis têm alguma importância para a avaliação de causalidade ou são essenciais na composição de um formulário de notificação; e iii) adicional - as variáveis foram consideradas não essenciais na avaliação de causalidade, mas supostamente incluídas por alguma outra utilidade prevista e disponibilidade de tempo e recursos para sua obtenção. Nesta etapa, também, foi quantificado o total de campos necessários para preenchimento em cada um dos formulários.

As variáveis consideradas vitais e complementares dos formulários ainda foram classificadas em cinco critérios, segundo sua contribuição para a avaliação de causalidade: i) relação temporal; ii) plausibilidade biológica ou farmacológica da reação adversa; iii) plausibilidade biológica ou farmacológica do medicamento; iv) binômio suspensão-reintrodução do medicamento; e, v) fator de explicação alternativo - possibilidade, em combinação ou isoladamente, de outra explicação para a reação adversa. A expressão "não se aplica" foi inserida nas variáveis que não se enquadraram em nenhum dos critérios.

$\mathrm{Na}$ última etapa, recorreu-se à análise de agrupamento hierárquico aglomerativo para identificar e separar os formulários similares em grupos diferentes, segundo a combinação de onze variáveis: a) total de pontos na composição dos itens considerados essenciais; b) total de campos a serem preenchidos nos formulários de cada país; c) total de variáveis consideradas vitais, complementares e adicionais; c) total de variáveis relacionadas com os critérios de avaliação de causalidade i, ii, iii, iv e v; e, d) total de variáveis classificadas como "não se aplica". De acordo com a análise de agrupamento, quanto mais próximo de zero mais similares são as unidades comparadas.

Um banco de dados foi organizado em planilha eletrônica (Microsoft Office Excel ${ }^{\circledR}$ ). Três variáveis de preenchimento automático pelo Notivisa-medicamento e relacionadas com a "identificação da notificação" foram excluídas de todas as análises.

As análises foram realizadas no software $\mathrm{R}$, versão 3.2.4 com o uso do pacote $\mathrm{R}$ comman- der $^{32}$. O método de normalização linear foi utilizado para a padronização das variáveis em uma mesma escala, com intervalo de 0 a 1 . A distância Euclidiana foi utilizada como medida de similaridade dos formulários e a agregação dos grupos diferentes foi realizada pelo método de Ward. A quantidade de agrupamentos foi definida automaticamente pelo pacote estatístico FactoMineR (Factor analysis and data mining with $R)^{33}$ a partir de um intervalo de agrupamento estabelecido como ótimo entre três e oito. $\mathrm{O}$ dendrograma foi a representação gráfica utilizada para interpretar os resultados da análise de agrupamento hierárquico aglomerativo.

Este estudo é parte de projeto de pesquisa aprovado pelo comité de ética em pesquisa do Hospital de Clínicas de Porto Alegre da Universidade Federal do Rio Grande do Sul.

\section{Resultados}

\section{Caracterização dos procedimentos necessários à notificação no Notivisa-medicamento}

Antes do acesso ao formulário eletrônico para notificação de suspeitas de RAM, há uma etapa anterior, cuja lógica de organização no Notivisa se inicia pela suposta "causa" do problema a ser notificado, aqui, o medicamento. Primeiramente, o Notivisa determina que o notificador identifique o produto-motivo da notificação a partir de uma lista de possibilidades oferecida à fonte notificadora, incluindo o medicamento. Logo em seguida, após a seleção da suposta causa (medicamento), há a necessidade de caracterização do "efeito" através da pergunta: "Houve danos à saúde?". Escolhendo a opção "sim" o sistema direciona para o preenchimento do formulário para notificação de suspeita de RAM, denominado, neste estudo, de Notivisa-medicamento.

O formulário do Notivisa-medicamento destinado ao profissional da saúde é composto por cinco domínios, totalizando 92 variáveis (excluindo as três referentes ao notificador). O último domínio, denominado de "Pendências", destina-se à informação de variáveis que não foram finalizadas e que devem ser corrigidas ou preenchidas para proceder o envio da notificação ao Notivisa-medicamento. Os demais domínios, em ordem que aparecem no formulário, são: i) evento adverso; ii) medicamentos/empresa; iii) paciente ou usuário; e, iv) outras informações (Tabela 1). 
Tabela 1. Caracterização do formulário eletrônico para notificação espontânea de suspeitas de RAM ${ }^{\star}$ do Notivisamedicamento.

\begin{tabular}{|c|c|c|c|}
\hline Domínio & $\begin{array}{c}\text { Total de } \\
\text { variáveis }\end{array}$ & $\begin{array}{l}\text { Tipo de preenchimento da } \\
\text { variável e } n^{\circ} \text { de variáveis (n) }\end{array}$ & $\begin{array}{c}\text { Tipo de variável e } \mathbf{n}^{\circ} \text { de } \\
\text { variáveis (n) }\end{array}$ \\
\hline Evento adverso & 19 & $\begin{array}{l}\text { Obrigatório; } \mathrm{n}=7 \\
\text { Parcialmente obrigatório; } \mathrm{n}=2 \\
\text { Não obrigatório; } \mathrm{n}=10\end{array}$ & $\begin{array}{l}\text { Aberta; } \mathrm{n}=8 \\
\text { Categórica; } \mathrm{n}=1 \\
\text { Formato de data; } \mathrm{n}=4 \\
\text { Lista com opções de respostas: pode } \\
\text { escolher apenas uma; } \mathrm{n}=5 \\
\text { Lista com opções de respostas: pode } \\
\text { escolher mais de uma; } \mathrm{n}=1\end{array}$ \\
\hline $\begin{array}{l}\text { Medicamentos/ } \\
\text { Empresa }\end{array}$ & 39 & $\begin{array}{l}\text { Obrigatório; } \mathrm{n}=4 \\
\text { Parcialmente obrigatório; } \mathrm{n}=5 \\
\text { Não obrigatório; } \mathrm{n}=30\end{array}$ & $\begin{array}{l}\text { Aberta; } \mathrm{n}=22 \\
\text { Categórica; } \mathrm{n}=6 \\
\text { Formato de data; } \mathrm{n}=5 \\
\text { Lista com opções de respostas: pode } \\
\text { escolher apenas uma; } \mathrm{n}=6\end{array}$ \\
\hline $\begin{array}{l}\text { Paciente ou } \\
\text { Usuário }\end{array}$ & 21 & $\begin{array}{l}\text { Obrigatório; } \mathrm{n}=11 \\
\text { Parcialmente obrigatório; } \mathrm{n}=3 \\
\text { Não obrigatório; } \mathrm{n}=7\end{array}$ & $\begin{array}{l}\text { Aberta; } \mathrm{n}=10 \\
\text { Categórica; } \mathrm{n}=7 \\
\text { Formato de data; } \mathrm{n}=1 \\
\text { Lista com opções de respostas: pode } \\
\text { escolher apenas uma; } \mathrm{n}=3\end{array}$ \\
\hline $\begin{array}{l}\text { Outras } \\
\text { informações }\end{array}$ & 13 & $\begin{array}{l}\text { Obrigatório; } \mathrm{n}=6 \\
\text { Não obrigatório; } \mathrm{n}=7\end{array}$ & $\begin{array}{l}\text { Aberta; } \mathrm{n}=3 \\
\text { Categórica; } \mathrm{n}=6 \\
\text { Lista com opções de respostas: pode } \\
\text { escolher apenas uma; } \mathrm{n}=1 \\
\text { Lista com opções de respostas: pode } \\
\text { escolher mais de uma; } \mathrm{n}=3\end{array}$ \\
\hline Total & 92 & $\begin{array}{l}\text { Obrigatório; } \mathrm{n}=28 \\
\text { Parcialmente obrigatório; } \mathrm{n}=10 \\
\text { Não obrigatório; } \mathrm{n}=54\end{array}$ & $\begin{array}{l}\text { Aberta; } \mathrm{n}=43 \\
\text { Categórica; } \mathrm{n}=20 \\
\text { Formato de data; } \mathrm{n}=10 \\
\text { Lista com opções de respostas: pode } \\
\text { escolher apenas uma; } \mathrm{n}=15 \\
\text { Lista com opções de respostas: pode } \\
\text { escolher mais de uma; } \mathrm{n}=4\end{array}$ \\
\hline
\end{tabular}
(RAM).

O domínio "Medicamentos/Empresa" apresentou o maior número de variáveis $(\mathrm{N}=39$; $42,4 \%)$ no formulário. As variáveis em formato "Aberta" (campo de texto livre) predominaram em relação aos demais tipos $(\mathrm{N}=43 ; 46,7 \%)$ (Tabela 1).

Algumas variáveis em que o preenchimento é obrigatório, conta com uma opção “ignorada”. Já as variáveis de preenchimento denominado parcialmente obrigatório são aquelas em que, para um conjunto de variáveis, o preenchimento é obrigatório, porém se enquadram em uma regra que não garante totalmente essa condição para algumas delas. Por exemplo, o preenchimento das variáveis "Data de nascimento", "Idade no momento do evento" e "Grupo de idade" é obrigatório apenas para uma dessas variáveis, ou seja, a fonte notificadora decide qual das variáveis poderá ser preenchida.

\section{Descrição do processo de avaliação de causalidade}

A partir da notificação, equipe de técnicos especialistas da Anvisa, geralmente composta por médicos e farmacêuticos, encarrega-se da avaliação de causalidade para cada par medicamento -reação adversa, visando determinar se há plausibilidade causal na relação entre o medicamento suspeito e a reação adversa notificada ou se apenas houve uma coincidência temporal, sendo a reação adversa ocasionada por outra causa ou um conjunto de causas que não necessariamente envolve o medicamento. Para tal, recebem trei- 
namentos referentes ao processo de farmacovigilância e avaliação da causalidade. Ao final do julgamento causal, o especialista classifica cada par medicamento-reação adversa contido em uma notificação em uma das categorias qualitativas de avaliação da causalidade proposta pela OMS, as quais são: definida, provável, possível, improvável, condicional e não classificada.

\section{Análise comparativa dos formulários dos países latino-americanos}

$\mathrm{Na}$ análise comparativa dos países estudados, o formulário da Venezuela obteve a maior pontuação (18 pontos), enquanto que o congênere da Guatemala alcançou a menor, registrando 8,5 pontos. Considerando os formulários dos $13 \mathrm{pa}$ íses, a mediana foi igual a 16 pontos e o formulário do Brasil alcançou 15 pontos (Tabela 2).
Todos os formulários estudados solicitaram informações sobre o paciente (idade e sexo), reação adversa, evolução do paciente, lista de medicamentos suspeitos, dose e frequência, data de início e de término de uso do medicamento(s) suspeito(s) e dados do notificador, totalizando sete dos 19 itens que foram considerados essenciais.

Apenas os formulários dos sistemas de farmacovigilância da Bolívia e Uruguai solicitaram, em um item à parte, informações sobre gravidez e lactação. A informação sobre "Conhecimento prévio de alguma alergia", na maioria dos formulários que contemplou este item, foi pedida juntamente com outros dados. A exceção ocorreu para o instrumento da Bolívia, que deu destaque a este item com duas indagações feitas de forma isolada: a) antecedentes de alergia em geral; e b) antecedentes de alergia a medicamentos.

Tabela 2. Itens considerados essenciais na notificação de reações adversas a medicamentos em formulários de países latinoamericanos.

\begin{tabular}{|c|c|c|c|c|c|c|c|c|c|c|c|c|c|}
\hline Itens essenciais & BR & AR & BO & $\mathrm{CH}$ & $\mathrm{CO}$ & CR & CU & GU & ME & PA & PE & UR & VE \\
\hline Informações do paciente & 1 & 1 & 1 & 1 & 1 & 1 & 1 & 1 & 1 & 1 & 1 & 1 & 1 \\
\hline Conhecimento prévio sobre alergia & 0 & 1 & 1 & 1 & 0 & 0 & 0 & 0 & 1 & 1 & 0 & 1 & 1 \\
\hline Condição de gravidez & 0 & 1 & 1 & 0 & 0 & 0 & 0 & 0 & 1 & 1 & 0 & 1 & 1 \\
\hline Diagnóstico clínico & 1 & 1 & 0 & 1 & 1 & 1 & 1 & 1 & 1 & 1 & 1 & 1 & 1 \\
\hline História relevante do paciente & 1 & 1 & 1 & 1 & 1 & 0 & 1 & 0 & 1 & 1 & 1 & 1 & 1 \\
\hline Informações da reação adversa & 1 & 1 & 1 & 1 & 1 & 1 & 1 & 1 & 1 & 1 & 1 & 1 & 1 \\
\hline Consequência da reação adversa & 1 & 1 & 1 & 1 & 1 & 0 & 1 & 0 & 0 & 1 & 0 & 1 & 1 \\
\hline Tratamento da reação adversa & 0 & 0 & 1 & 1 & 1 & 0 & 1 & 0 & 0 & 0 & 0 & 1 & 1 \\
\hline $\begin{array}{l}\text { Evolução do paciente sobre a reação } \\
\text { adversa }\end{array}$ & 1 & 1 & 1 & 1 & 1 & 1 & 1 & 1 & 1 & 1 & 1 & 1 & 1 \\
\hline Lista de medicamentos suspeitos & 1 & 1 & 1 & 1 & 1 & 1 & 1 & 1 & 1 & 1 & 1 & 1 & 1 \\
\hline $\begin{array}{l}\text { Dose e frequência do } \\
\text { medicamento(s) suspeito(s) }\end{array}$ & 1 & 1 & 1 & 1 & 1 & 1 & 1 & 1 & 1 & 1 & 1 & 1 & 1 \\
\hline $\begin{array}{l}\text { Data de início e término do } \\
\text { medicamento(s) suspeito(s) }\end{array}$ & 1 & 1 & 1 & 1 & 1 & 1 & 1 & 1 & 1 & 1 & 1 & 1 & 1 \\
\hline $\begin{array}{l}\text { Número do lote e data de validade } \\
\text { do medicamento(s) suspeito(s) }\end{array}$ & 1 & 1 & 0,5 & 0,5 & 1 & 0,5 & 0,5 & 0,5 & 1 & 0 & 0,5 & 1 & 1 \\
\hline $\begin{array}{l}\text { Lista de medicamentos } \\
\text { concomitantes }\end{array}$ & 1 & 1 & 1 & 1 & 1 & 0 & 1 & 0 & 1 & 1 & 1 & 1 & 1 \\
\hline Ações adotadas & 1 & 0 & 0 & 1 & 0 & 0 & 0 & 0 & 1 & 1 & 0 & 0 & 1 \\
\hline Suspensão do uso do medicamento & 1 & 1 & 1 & 1 & 1 & 1 & 1 & 0 & 1 & 1 & 0 & 0 & 1 \\
\hline $\begin{array}{l}\text { Reintrodução do medicamento } \\
\text { suspeito }\end{array}$ & 1 & 1 & 0 & 1 & 1 & 1 & 1 & 0 & 1 & 1 & 0 & 1 & 1 \\
\hline Causalidade & 0 & 0 & 1 & 0 & 1 & 0 & 0 & 0 & 0 & 0 & 0 & 0 & 0 \\
\hline Dados do notificador & 1 & 1 & 1 & 1 & 1 & 1 & 1 & 1 & 1 & 1 & 1 & 1 & 1 \\
\hline Total & 15 & 16 & 15,5 & 16,5 & 16 & 10,5 & 14,5 & 8,5 & 16 & 16 & 10,5 & 16 & 18 \\
\hline
\end{tabular}


O requisito de preenchimento de informação referente ao diagnóstico clínico foi verificado em dez países como "Motivo da prescrição/indicação”. O formulário da Argentina solicita "Diagnóstico presuntivo". O brasileiro requer a "Indicação terapêutica”. Não foi identificada nenhuma menção esclarecedora a este item no instrumento da Bolívia. Outro item que apresentou heterogeneidade nos formulários dos países foi "História relevante do paciente", sendo comuns as expressões, como "Patologia de base", "Antecedentes importantes/relevantes" e "História clínica".

Os formulários da Costa Rica e Guatemala não explicitaram de forma clara o pedido de informações sobre uso de medicamentos concomitantes. Bolívia, Chile, Costa Rica, Cuba, Guatemala e Peru solicitaram informações apenas do número de lote do medicamento em investigação, levando a receber, cada um deles, 0,5 pontos (Tabela 2). O item "Ações adotadas decorrentes da reação adversa" consta em seis formulários no formato de perguntas e junto dos itens relacionados ao binômio suspensão-reintrodução do medicamento. Os instrumentos da Bolívia e da Colômbia foram os únicos que pediram uma classificação de causalidade para a notificação com o uso das categorias da OMS (Tabela 2 ).

A mediana das variáveis para preenchimento no formulário dos países foi de 41, variando de 26 (Guatemala) a 95 (Brasil). O número de variáveis destinadas à identificação da fonte notificadora variou de 2 (Cuba e México) até 8 (Argentina e Chile), com mediana igual a 4 .

A Tabela 3 apresenta uma caracterização dos formulários de notificação dos países. Em todos, a categoria "Vital" obteve o maior número de variáveis a serem preenchidas, sendo que o formulário panamenho registrou o maior percentual $(86,7 \%)$ e o brasileiro, o menor $(47,8 \%)$. Em relação às variáveis definidas como vitais e complementares, a maior parte contribuiu na avaliação de causalidade em farmacovigilância, como fator de explicação alternativo em todos os formulários analisados, variando de $38,1 \%$ (Costa Rica) a 64,6\% (Bolívia) (Tabela 3).

A partir do agrupamento hierárquico representado pelo dendrograma (Figura 1) é possível observar que os formulários mais similares entre si são o da Costa Rica e o da Guatemala, seguido do Peru (agrupamento 4), pois apresentaram uma altura mais próxima de zero. Todos os formulários ficaram a uma altura inferior a 2 , à exceção do instrumento do Brasil (agrupamento 1) que se apresentou como o mais dissimilar, registrando uma altura de aproximadamente 3,5 (Figura 1).

\section{Discussão}

Este estudo avaliou comparativamente o formulário do Notivisa-medicamento e de outros doze países latino-americanos como um instrumento de coleta de informações para subsidiar o monitoramento e a avaliação em sistemas governamentais de farmacovigilância. Os formulários dos países estudados variaram em relação aos itens considerados essenciais para uma adequada notificação de suspeitas de RAM, quantidade de variáveis a serem preenchidas e categorias e critérios importantes para uma apropriada avaliação de causalidade. Especificamente, em relação aos itens considerados essenciais, essa heterogeneidade também foi verificada em artigo publicado por Bandekar et al. ${ }^{11}$, que analisaram 10 países diferentes dos que foram avaliados neste estudo.

A diversidade das características dos formulários foi constatada no dendrograma que apresentou dois agrupamentos formados cada um por um único país. Apesar disso, observou-se uma similaridade de dois agrupamentos, envolvendo, oito países (agrupamento 2) e três países (agrupamento 4). O agrupamento 2, que obteve o maior número de formulários similares, está relacionado principalmente com as variáveis: total de pontos na composição dos itens considerados essenciais e total de variáveis relacionadas com os critérios de avaliação de causalidade iii e iv. As demais variáveis estão relacionadas basicamente com o agrupamento 1.

Singh e Bhatt ${ }^{12}$ propuseram um formulário genérico para a notificação de RAM com 58 variáveis, incluído cinco sobre dados da fonte notificadora. O formulário do Brasil, entre todos os países estudados, foi o que apresentou o maior número de variáveis para preenchimento, ultrapassando em $63,8 \%$ o montante proposto pelo formulário de Singh e Bhatt ${ }^{12}$. Algumas das variáveis de importância para a avaliação de causalidade que constavam explicitamente no formulário genérico e não no formulário do Brasil foram: alergia, gravidez, hábitos, como consumo de álcool e tabaco, disfunção hepática/renal e data de recuperação do paciente ${ }^{12}$.

Constatou-se explicitamente a solicitação das informações referentes à alergia e gravidez apenas nos formulários de sete e de seis países latino-americanos, respectivamente, quesitos relevantes para a segurança de uso e avaliação de causalidade ${ }^{11}$.

Apenas o formulário da Bolívia não solicitou informações sobre o diagnóstico clínico do paciente. Algumas RAM apresentam um padrão 
Tabela 3. Caracterização dos formulários de notificação de reações adversas a medicamentos dos países latinoamericanos.

\begin{tabular}{|c|c|c|c|c|c|c|c|c|c|c|}
\hline \multirow{2}{*}{ País } & \multicolumn{4}{|c|}{$\begin{array}{l}\text { Categorias de avaliação } \\
\text { de causalidade }\end{array}$} & \multicolumn{6}{|c|}{$\begin{array}{c}\text { Critérios que contribuem para avaliação } \\
\text { de causalidade }\end{array}$} \\
\hline & $\begin{array}{c}\mathrm{Vi} \\
(\%)\end{array}$ & $\begin{array}{c}\text { Co } \\
(\%)\end{array}$ & $\begin{array}{l}\text { Ad } \\
(\%)\end{array}$ & Total & $\begin{array}{c}\mathrm{i} \\
(\%)\end{array}$ & $\begin{array}{c}\text { ii } \\
(\%)\end{array}$ & $\begin{array}{c}\text { iii } \\
(\%)\end{array}$ & $\begin{array}{c}\text { iv } \\
(\%)\end{array}$ & $\begin{array}{c}\mathbf{v} \\
(\%)\end{array}$ & $\begin{array}{c}\text { vi } \\
(\%)\end{array}$ \\
\hline Brasil & $\begin{array}{c}44 \\
(47,8)\end{array}$ & $\begin{array}{c}19 \\
(20,7)\end{array}$ & $\begin{array}{c}29 \\
(31,5)\end{array}$ & 92 & $\begin{array}{c}12 \\
(19,1)\end{array}$ & $\begin{array}{c}4 \\
(6,3)\end{array}$ & $\begin{array}{c}3 \\
(4,8)\end{array}$ & $\begin{array}{c}5 \\
(7,9)\end{array}$ & $\begin{array}{c}29 \\
(46,0)\end{array}$ & $\begin{array}{c}10 \\
(15,9)\end{array}$ \\
\hline Argentina & $\begin{array}{c}17 \\
(65,4)\end{array}$ & $\begin{array}{c}5 \\
(19,2)\end{array}$ & $\begin{array}{c}4 \\
(15,4)\end{array}$ & 26 & $\begin{array}{c}4 \\
(18,2)\end{array}$ & $\begin{array}{c}1 \\
(4,5)\end{array}$ & $\begin{array}{c}2 \\
(9,1)\end{array}$ & $\begin{array}{c}2 \\
(9,1)\end{array}$ & $\begin{array}{c}10 \\
(45,5)\end{array}$ & $\begin{array}{c}3 \\
(13,6)\end{array}$ \\
\hline Bolívia & $\begin{array}{c}39 \\
(66,1)\end{array}$ & $\begin{array}{c}9 \\
(15,3)\end{array}$ & $\begin{array}{c}11 \\
(18,6)\end{array}$ & 59 & $\begin{array}{c}8 \\
(16,6)\end{array}$ & $\begin{array}{c}2 \\
(4,2)\end{array}$ & $\begin{array}{c}1 \\
(2,1)\end{array}$ & $\begin{array}{c}1 \\
(2,1)\end{array}$ & $\begin{array}{c}31 \\
(64,6)\end{array}$ & $\begin{array}{c}5 \\
(10,4)\end{array}$ \\
\hline Chile & $\begin{array}{c}31 \\
(79,5)\end{array}$ & $\begin{array}{c}5 \\
(12,8)\end{array}$ & $\begin{array}{c}3 \\
(7,7)\end{array}$ & 39 & $\begin{array}{c}6 \\
(16,6)\end{array}$ & $\begin{array}{c}1 \\
(2,8)\end{array}$ & $\begin{array}{c}2 \\
(5,5)\end{array}$ & $\begin{array}{c}4 \\
(11,1)\end{array}$ & $\begin{array}{c}16 \\
(44,4)\end{array}$ & $\begin{array}{c}9 \\
(25,0)\end{array}$ \\
\hline Colômbia & $\begin{array}{c}27 \\
(61,4)\end{array}$ & $\begin{array}{c}5 \\
(11,4)\end{array}$ & $\begin{array}{c}12 \\
(27,2)\end{array}$ & 44 & $\begin{array}{c}4 \\
(12,5)\end{array}$ & $\begin{array}{c}2 \\
(6,3)\end{array}$ & $\begin{array}{c}2 \\
(6,3)\end{array}$ & $\begin{array}{c}6 \\
(18,7)\end{array}$ & $\begin{array}{c}13 \\
(40,6)\end{array}$ & $\begin{array}{c}5 \\
(15,6)\end{array}$ \\
\hline Costa Rica & $\begin{array}{c}16 \\
(66,7)\end{array}$ & $\begin{array}{c}5 \\
(20,8)\end{array}$ & $\begin{array}{c}3 \\
(12,5)\end{array}$ & 24 & $\begin{array}{c}4 \\
(19,0)\end{array}$ & $\begin{array}{c}1 \\
(4,8)\end{array}$ & $\begin{array}{c}1 \\
(4,8)\end{array}$ & $\begin{array}{c}3 \\
(14,3)\end{array}$ & $\begin{array}{c}8 \\
(38,1)\end{array}$ & $\begin{array}{c}4 \\
(19,0)\end{array}$ \\
\hline Cuba & $\begin{array}{c}18 \\
(60,0)\end{array}$ & $\begin{array}{c}8 \\
(26,7)\end{array}$ & $\begin{array}{c}4 \\
(13,3)\end{array}$ & 30 & $\begin{array}{c}4 \\
(15,4)\end{array}$ & $\begin{array}{c}1 \\
(3,8)\end{array}$ & $\begin{array}{c}1 \\
(3,8)\end{array}$ & $\begin{array}{c}4 \\
(15,4)\end{array}$ & $\begin{array}{c}10 \\
(38,5)\end{array}$ & $\begin{array}{c}6 \\
(23,1)\end{array}$ \\
\hline Guatemala & $\begin{array}{c}12 \\
(60,0)\end{array}$ & $\begin{array}{c}6 \\
(30,0)\end{array}$ & $\begin{array}{c}2 \\
(10,0)\end{array}$ & 30 & $\begin{array}{c}4 \\
(22,2)\end{array}$ & $\begin{array}{c}1 \\
(5,6)\end{array}$ & $\begin{array}{c}1 \\
(5,6)\end{array}$ & $\begin{array}{c}0 \\
(0,0)\end{array}$ & $\begin{array}{c}8 \\
(44,4)\end{array}$ & $\begin{array}{c}4 \\
(22,2)\end{array}$ \\
\hline México & $\begin{array}{c}28 \\
(71,8)\end{array}$ & $\begin{array}{c}5 \\
(12,8)\end{array}$ & $\begin{array}{c}6 \\
(15,4)\end{array}$ & 39 & $\begin{array}{c}5 \\
(15,1)\end{array}$ & $\begin{array}{c}1 \\
(3,0)\end{array}$ & $\begin{array}{c}2 \\
(6,2)\end{array}$ & $\begin{array}{c}8 \\
(24,0)\end{array}$ & $\begin{array}{c}15 \\
(45,5)\end{array}$ & $\begin{array}{c}2 \\
(6,2)\end{array}$ \\
\hline Panamá & $\begin{array}{c}26 \\
(86,7\end{array}$ & $\begin{array}{c}3 \\
(10,0)\end{array}$ & $\begin{array}{c}1 \\
(3,3)\end{array}$ & 30 & $\begin{array}{c}6 \\
(20,7)\end{array}$ & $\begin{array}{c}1 \\
(3,4)\end{array}$ & $\begin{array}{c}1 \\
(3,4)\end{array}$ & $\begin{array}{c}6 \\
(20,7)\end{array}$ & $\begin{array}{c}13 \\
(44,8)\end{array}$ & $\begin{array}{c}2 \\
(7,0)\end{array}$ \\
\hline Peru & $\begin{array}{c}19 \\
(73,1)\end{array}$ & $\begin{array}{c}5 \\
(19,2)\end{array}$ & $\begin{array}{c}2 \\
(7,7)\end{array}$ & 26 & $\begin{array}{c}6 \\
(25,0)\end{array}$ & $\begin{array}{c}1 \\
(4,2)\end{array}$ & $\begin{array}{c}1 \\
(4,2)\end{array}$ & $\begin{array}{c}0 \\
(0,0)\end{array}$ & $\begin{array}{c}13 \\
(54,1)\end{array}$ & $\begin{array}{c}3 \\
(12,5)\end{array}$ \\
\hline Uruguai & $\begin{array}{c}25 \\
(62,5)\end{array}$ & $\begin{array}{c}6 \\
(15,0)\end{array}$ & $\begin{array}{c}9 \\
(22,5)\end{array}$ & 40 & $\begin{array}{c}6 \\
(19,3)\end{array}$ & $\begin{array}{c}1 \\
(3,2)\end{array}$ & $\begin{array}{c}1 \\
(3,2)\end{array}$ & $\begin{array}{c}1 \\
(3,2)\end{array}$ & $\begin{array}{c}18 \\
(58,1)\end{array}$ & $\begin{array}{c}5 \\
(16,1)\end{array}$ \\
\hline Venezuela & $\begin{array}{c}24 \\
(61,5)\end{array}$ & $\begin{array}{c}9 \\
(23,1)\end{array}$ & $\begin{array}{c}6 \\
(15,4)\end{array}$ & 39 & $\begin{array}{c}6 \\
(18,2)\end{array}$ & $\begin{array}{c}1 \\
(3,0)\end{array}$ & $\begin{array}{c}3 \\
(9,1)\end{array}$ & $\begin{array}{c}2 \\
(6,1)\end{array}$ & $\begin{array}{c}16 \\
(48,5)\end{array}$ & $\begin{array}{c}6 \\
(18,2)\end{array}$ \\
\hline
\end{tabular}

Notas: Vi - vital; Co - Complementar; e Ad - Adicional.

i) relação temporal; ii) plausibilidade biológica ou farmacológica da reação adversa; iii) plausibilidade biológica ou farmacológica do medicamento; iv) binômio suspensão-reintrodução do medicamento; v) fator de explicação alternativo; e vi) não se aplica.

clínico clássico de sinais e sintomas que podem diferenciar de certas enfermidades. Por exemplo, as reações adversas designadas como necrose epidérmica tóxica e aplasia de medula óssea são entidades distintas que normalmente são reconhecidas como RAM pelo médico ${ }^{34}$. Esta determinação causal definida pelo médico facilita o processo de avaliação de causalidade em farmacovigilância. A ausência de itens essenciais no formulário de notificação pode comprometer a avaliação de causalidade individual, gerando sinais de segurança equivocados.

A dificuldade na separação entre RAM e doença de base (ou outra condição fisiológica e psicossocial) para a qual o medicamento foi prescrito favorece a presença de outras explicações alternativas na avaliação de causalidade ${ }^{35}$. A título exemplificativo, um episódio hipotensivo grave e abrupto pode ser o fator causador da insuficiência hepática aguda ao invés do medicamento utilizado pelo paciente ${ }^{36}$. Essa questão reforça a importância da necessidade de informação sobre o diagnóstico médico, bem como sobre histórias clínicas e psicossociais pregressas do paciente nos formulários.

Se um medicamento foi administrado antes do início de uma reação adversa essa sequência temporal é suficiente para que uma fonte notificadora suspeite de uma relação causal, procedendo, assim, o registro da notificação nos sistemas de farmacovigilância de cada país. Os formulários estudados apresentam diversas variáveis que contemplam esse aspecto importante para a avaliação de causalidade, como datas de início 


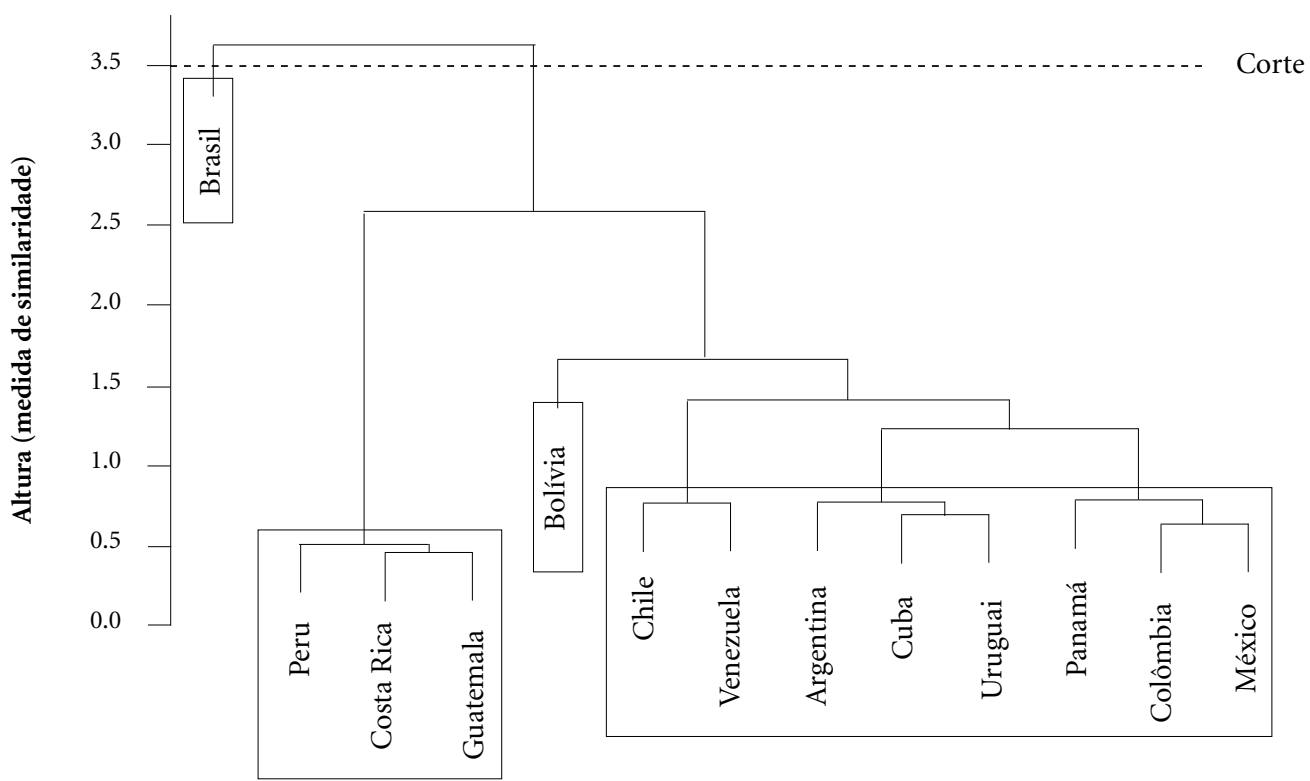

Agrupamento $1=$ Brasil

Agrupamento 2 = Argentina, Chile, Colômbia, Cuba, México, Panamá, Uruguai e Venezuela Agrupamento 3 = Bolívia

Agrupamento 4 - Costa Rica, Guatemala e Peru

Figura 1. Dendrograma dos formulários de notificação de suspeitas de reações adversas a medicamentos dos países latinoamericanos.

e término da reação adversa e de uso do medicamento suspeito. O momento do surgimento da RAM após o início do uso do medicamento pode fornecer pistas sobre o possível mecanismo clínico-patológico (por exemplo, curta latência: anafilaxia; longa latência: cirrose $)^{36}$. No entanto, muitas das notificações registradas nos sistemas de farmacovigilância representam apenas uma associação meramente temporal ${ }^{37}$, cujo medicamento administrado não é a causa do problema notificado.

Todos os formulários dos países contemplaram informações referentes à dose administrada e frequência de uso, itens essenciais para a avaliação de causalidade quanto à dose e/ou frequência inapropriadas do medicamento, diferenciando -a de errônea, interpretação como RAM, e sim como erro de medicação ${ }^{11}$.

A informação sobre a data de validade do medicamento foi constatada no formulário de seis países, aspecto essencial para adequada identificação do medicamento suspeito ${ }^{38}$, podendo ser considerada também um fator de explicação alternativo para a ocorrência de inefetividade terapêutica e não de reação adversa.

O peso e a altura do paciente são duas variáveis importantes na avaliação de causalidade de uma RAM, pois ambas são utilizadas para calcular o índice de massa corporal e área da superfície do corpo. Tais medidas ajudam na determinação da dosagem apropriada de um medicamento, especialmente naqueles com baixo índice terapêutico ${ }^{12}$, e, consequentemente, na distinção entre reação adversa e intoxicação medicamentosa. Ambas variáveis se fizeram presentes nos formulários do Brasil, Bolívia, Chile, Colômbia, México, Panamá e Venezuela e foram classificadas como vitais na avaliação de causalidade em farmacovigilância.

Outra variável especial é a raça/cor/etnia, que em algumas situações é considerada como fator de risco para o aparecimento de $\mathrm{RAM}^{12}$. Esta variável foi verificada apenas nos formulários da Brasil, Chile e Colômbia. Igualmente importante 
foi a constatação da ausência de itens essenciais nos formulários de alguns países, envolvendo potenciais fatores alternativos de explicação, como consumo de álcool e tabaco, condição que impede a exclusão desses fatores do processo de análise e interpretação do julgamento causal.

Um importante fator de explicação alternativo presente na investigação de causalidade é o uso concomitante de medicamentos. No caso do efeito dos antipsicóticos de segunda geração no ganho de peso, por exemplo, a utilização de outro fármaco para perda de peso poderá obscurecer ou confundir o julgamento causal ${ }^{39}$. Ademais, o aparecimento da reação adversa pode ter sido uma consequência de interação medicamentosa entre medicamento suspeito e congêneres em uso concomitante. Informações sobre o uso de medicamentos concomitantes foram identificadas em formulário de 11 países latino-americanos. O formulário do Brasil solicita à fonte notificadora que indique ainda se houve alguma suspeita de interação medicamentosa, assim como o da Venezuela.

Alguns dos itens essenciais na avaliação de causalidade em farmacovigilância incluem informações sobre suspensão e reintrodução do medicamento suspeito ${ }^{11}$. Se a reação adversa é resolvida com a retirada do fármaco e ocorre novamente com a reintrodução, então, o binômio suspensão-reintrodução é considerado como positivo e caracterizado como forte evidência de envolvimento do medicamento com a reação adversa $^{36}$. Exemplo disso foi o sinal de segurança gerado a partir das notificações de uma série de casos de pacientes com arritmias graves após uso de cisaprida, que incluiu evidências de reicidiva com a reintrodução do fármaco ${ }^{40}$. Essas informações não foram solicitadas nos formulários da Bolívia, Guatemala e Peru. Devido a questões éticas e legais, dados sobre a reintrodução do medicamento podem não estar disponíveis para registro no formulário. Ademais, mesmo em determinados contextos de avaliação do benefício-risco para as RAM mais conhecidas e graves, a reintrodução do fármaco não deve ser, intencionalmente, reali$\mathrm{zada}^{36}$. No entanto, a reintrodução acidental justifica a necessidade da informação no formulário.

As fontes notificadoras dos formulários de nove países são solicitadas a indicar a consequência da reação adversa para o paciente, em termos de gravidade do problema (por exemplo, motivou hospitalização, anomalia congênita e óbito). Algum grau de viés de classificação é inerente à distinção de reação caracterizada como grave e não grave. Por exemplo, as notificações de reação no local da injeção, geralmente, não são de grande importância clínica, mas podem ser classificadas como graves se resultarem em uma hospitalização ainda que breve. Inversamente, alguns relatos de eventos que são clinicamente importantes, tais como a paralisia de Bell, podem não ser classificados como graves, porque não resultaram em uma internação hospitalar ${ }^{37}$.

Os instrumentos de notificação da Bolívia e da Colômbia solicitam às fontes notificadoras uma classificação de causalidade global. Para os autores deste estudo, a avaliação de causalidade deve ser feita para cada par medicamento suspeito-reação adversa, cuja imputação de causalidade deve considerar ao menos todas as informações disponíveis no formulário. Ambos os países adotaram o método de avaliação de causalidade proposto pela $\mathrm{OMS}^{14}$ e, especificamente, o formulário do sistema colombiano de farmacovigilância apresenta um campo a mais, do tipo texto livre, para a "justificação da análise de acordo a causalidade".

A OMS sustenta que um modelo único de formulário corre o risco de ficar aquém ou "demasiado ambicioso", dependendo das circunstâncias e necessidades de cada país ${ }^{41}$. Uma possível solução para permitir uma maior flexibilidade em vários contextos seria desenvolver um sistema hierárquico, prevendo modelo mínimo de informação seguido de outros níveis de modelos intermediários e mais completos ${ }^{41}$.

$\mathrm{O}$ julgamento causal em farmacovigilância é um processo complexo, difícil e bastante desafiador. Envolve a inclusão ou a exclusão, de forma combinada ou isoladamente, de explicações alternativas e a caracterização da presença de potenciais vieses que influenciam a validade de estudos sobre causalidade em farmacoepidemio$\operatorname{logia}^{42}$. A maioria das variáveis vitais e complementares nos formulários estudados contribuiram como fatores de explicação alternativos, o que demonstra uma preocupação com esse tipo de erro na avaliação de causalidade feita por técnicos dos órgãos encarregados das atividades de farmacovigilância em cada país.

A principal limitação deste estudo está relacionada à possibilidade de viés de seleção dos formulários dos diferentes países, pois a obtenção deles, à exceção do brasileiro, ocorreu por meio de websites oficiais dos governos que poderiam estar desatualizados. Além disso, pode ter havido discrepância entre os processos de notificação, registro, análise, interpretação e avaliação de causalidade e a correspondência dos mesmos nos formulários utilizados. A pontuação obtida para cada formulário pode estar subestimada, 
uma vez que os autores se debruçaram em analisar apenas os itens que estavam explicitamente referidos no instrumento de notificação. Os autores não checaram as instruções de preenchimento dos formulários.

Outra limitação foi assumir que todos os formulários analisados são utilizados por profissionais da saúde. Esta questão não pode ser totalmente esclarecida nos formulários da Bolívia, Colômbia, Costa Rica, Guatemala e Uruguai, muito embora, não há nenhuma informação que mencione o impedimento de uso por parte dos profissionais da saúde.

Observou-se, também, que alguns formulários não são dedicados exclusivamente à notificação de suspeitas de RAM, como é o caso do Brasil, Costa Rica, Guatemala e Venezuela, que aceitam a notificação de outro tipo de evento adverso, por exemplo, inefetividade terapêutica. Esta estratégia poderá comprometer a coleta, a análise e a interpretação dos dados de um ou do outro evento a ser notificado. Isto, talvez, explique o excesso de dados encontrados, particularmente, no formulário do Brasil. O excesso de dados e informações solicitados pelo formulário do Brasil podem dificultar a avaliação de causalidade individual e, também, limitar o desempenho do SINAF, no que tange aos seus atributos oportunidade, simplicidade e aceitabilidade ${ }^{43,44}$.

No que tange ao sistema de farmacovigilância do Brasil, em 10 de dezembro de 2018, deu-se início a um novo sistema eletrônico para a notificação de eventos adversos a medicamentos e vacinas, denominado de Vigimed - versão adaptada do sistema VigiFlow, oferecido pela OMS aos Centros Nacionais que fazem parte do Programa Internacional para o Monitoramento de Medicamentos $^{45}$. A transição entre os sistemas Notivisa-medicamento e VigiMed está ocorrendo gradualmente com as habilitações dos ambientes de notificação destinados aos cidadãos (usuários de medicamentos), profissionais de saúde, instituições de saúde, incluindo a Rede sentinela e detentores de registro de medicamento no país ${ }^{45}$.

Os achados do presente estudo permitem sugerir diferenças entre os formulários em relação a aspectos considerados como essenciais e vitais em farmacovigilância, sobretudo quanto às informações necessárias para o processo de caracterização da causalidade medicamento-reação adversa. Bailey et al. ${ }^{46}$ encontraram um alto grau de variabilidade nos campos de dados utilizados para relatar eventos adversos a medicamentos em 100 sistemas de farmacovigilância, com abrangência internacional, nacional e local. Tais resultados limitam a comparabilidade dos dados gerados nos diferentes sistemas e comprometem os esforços para agrupar e analisar dados entre coortes, para melhor detecção de sinais de segurança raros e emergentes ${ }^{46}$.

A harmonização de dados para relatar suspeitas de RAM pode ser útil no processo contínuo e sistemático de aperfeiçoamento dos sistemas de informação de farmacovigilância de diferentes países, permitindo dessa maneira que futuros estudos procedam não apenas em avaliações comparativas mais aprofundadas como também avaliando a qualidade dos dados e dos sistemas nacionais de farmacovigilância. Tais sistemas de informação pertencem aos mecanismos de comunicação entre os diferentes atores envolvidos no uso seguro de medicamentos, compondo, dessa maneira, a estrutura de farmacogovernança dos países ${ }^{15}$. Nesta direção, é possível presumir que os formulários avaliados representem de alguma maneira, ainda que sinteticamente, a intencionalidade dos processos de trabalho praticados pelos órgãos de farmacovigilância implicados na investigação das reações e, consequentemente, o estabelecimento de inferências de causalidade.

\section{Colaboradores}

DM Mota, A Vigo e RS Kuchenbecker participaram na concepção e desenho do estudo, análise e interpretação dos dados do manuscrito. DM Mota elaborou a redação do artigo e A Vigo e RS Kuchenbecker participaram da sua revisão crítica e aprovação final. 


\section{Referências}

1. Souza TT de, Godoy RR, Rotta I, Pontarolo R, Fernandez-Llimos F, Correr CJ. Morbidade e mortalidade relacionadas a medicamentos no Brasil: revisão sistemática de estudos observacionais. Rev Ciênc Farm Básica Apl 2014; 35(4):519-532.

2. Kongkaew C, Noyce P, Ashcroft D. Hospital admissions associated with adverse drug reactions: a systematic review of prospective observational studies. Ann Pharmacother 2008; 42(7):1017-25.

3. Mota DM, Melo JRR, Freitas DRC, Machado M. Perfil da mortalidade por intoxicação com medicamentos no Brasil, 1996-2005: retrato de uma década. Cien Saude Colet 2012; 17(1):61-70.

4. Patel H, Bell D, Molokhia M, Srishanmuganathan J, Patel M, Car J, Majeed A. Trends in hospital admissions for adverse drug reactions in England: analysis of national hospital episode statistics 1998-2005. BMC Clin Pharmacol 2007; 7:9.

5. Lazarou J, Pomeranz BH, Corey PN. Incidence of Adverse Drug Reactions in Hospitalized Patients. J Am Med Assoc 1998; 279(15):1200.

6. Agência Nacional de Vigilância Sanitária (Anvisa). Guias de farmacovigilância para detentores de registro de medicamentos [Internet]. Brasília: Anvisa; 2010. [cited 2016 Mar 16]. Available from: http://portal.anvisa. gov.br/wps/wcm/connect/e687bc0047457e328a38de$3 \mathrm{fbc4c6735/Guias+de+Farmacovigilância+Detento-}$ res+Registro+Medicamento.pdf?MOD=AJPERES

7. Carnovale C, Brusadelli T, Zuccotti G, Beretta S, Sullo MG, Capuano A, Rossi F, Moschini M, Mugelli A, Vannacci A, Laterza M, Clementi E, Radice S, MEAP Group. The importance of monitoring adverse drug reactions in pediatric patients: the results of a national surveillance program in Italy. Expert Opin Drug Saf 2014; 13(Supl. 1):1-8.

8. Venulet J, ten Ham M. Methods for monitoring and documenting adverse drug reactions. Int J Clin Pharmacol Ther 1996; 34(3):112-129.

9. Sahu RK, Yadav R, Prasad P, Roy A, Chandrakar S. Adverse drug reactions monitoring: prospects and impending challenges for pharmacovigilance. Springerplus 2014; 3(1):695.

10. Martins ACM. Eventos adversos a medicamentos: bancos de dados administrativos de pacientes hospitalizados e registro de óbitos como fonte de informação. Rio de Janeiro: Escola Nacional de Saúde Pública; 2015.

11. Bandekar MS, Anwikar SR, Kshirsagar NA. Quality check of spontaneous adverse drug reaction reporting forms of different countries. Pharmacoepidemiol Drug Saf 2010; 19(11):1181-1185.

12. Singh A, Bhatt P. Comparative evaluation of adverse drug reaction reporting forms for introduction of a spontaneous generic ADR form. J Pharmacol Pharmacother 2012; 3(3):228.

13. Agência Nacional de Vigilância Sanitária (Anvisa). Diretrizes para o Gerenciamento do Risco em Farmacovigilância. Brasília: Anvisa; 2008.
14. World Health Organization (WHO). The use of the WHO-UMC system for standardized case causality assessment. Uppsala: The Uppsala Monitoring Centre; 2005.

15. Moscou K, Kohler JC, MaGahan A. Governance and pharmacovigilance in Brazil: a scoping review. J Pharm Policy Pract. J Pharm Policy Pract 2016; 9:3.

16. World Health Organization (WHO). Uppsala Monitoring Centre [Internet]. 2016 [cited 2016 Mar 16]. Available from: http://www.who-umc.org/DynPage. aspx $? \mathrm{id}=100653 \& \mathrm{mn} 1=7347 \& \mathrm{mn} 2=7252 \& \mathrm{~m}-$ $\mathrm{n} 3=7322 \& \mathrm{mn} 4=7442$

17. Argentina. Administración Nacional de Medicamentos A y TM. Sistema Nacional de Farmacovigilancia - Comunicación de Eventos Adversos de Medicamentos [Internet]. [cited 2015 Dec 28]. Available from: http:// sistemas.anmat.gov.ar/aplicaciones_net/applications/ fvg_eventos_adversos_nuevo/index.html

18. Bolivia. Ministerio de la Salud y Deportes. Sistema Nacional de Farmacovigilancia [Internet]. [cited 2015 Dec 30]. Available from: http://unimed.minsalud.gob. bo/fvigilancia/snfv.htm\#

19. Chile. Ministerio de la Salud. Formulario de Notificacion de Reacción Adversa a Medicamentos (RAM) [Internet]. [cited 2015 Dec 30]. Available from: http:// www.ispch.cl/anamed/subdeptofarmacovigilancia/ notificacion_ram

20. Colômbia. Instituto Nacional de Vigilancia de Medicamentos y Alimentos. Formato reporte de sospecha de eventos adversos a medicamentos - FOREAM [Internet]. [cited 2015 Dec 30]. Available from: https:// www.invima.gov.co/index.php?option=com_content\&view=article\&id=753:reporte-de-reacciones-adversas\&catid $=191$ : farmacovigilancia\&Itemid $=335$

21. Rica. Ministerio de la Salud. Formulario: Notificación de sospecha de reacción adversa a un medicamento (Tarjeta Amarilla) [Internet]. 2013 [cited 2015 Dec 30]. Available from: http://www.ministeriodesalud. go.cr/index.php/formularios-de-notificacion-RAM

22. Cuba. Centro Estatal para el Controle de Medicamentos Equipos y Dispositivos Médicos. Modelo Reporte de RAM Profesionales Sanitarios [Internet]. [cited 2015 Dec 30]. Available from: http://www.cecmed.cu/farmacovigilancia/profesionales

23. Guatemala. Govierno de la Republica. Boleta de notificación espontanea de sospecha de reacción adversa y problemas relacionados con medicamentos y Biológicos [Internet]. [cited 2016 Mar 17]. Available from: http:// www.mspas.gob.gt/index.php/en/servicios-de-farmacovigilancia.html

24. México. Secretaría de Salud. Comisión Federal para la Protección contra Riesgos Sanitarios. Aviso de sospechas de reacciones adversas de medicamentos [Internet]. [cited 2015 Dec 30]. Available from: http://www. cofepris.gob.mx/AZ/Paginas/Farmacovigilancia/Formato-para-el-informe-de-sospechas-de-reacciones -adversas-de-los-medicamentos.aspx 
25. Panamá. Governo de la Republica. Formulario de Notificación por Sospecha de Reacciones Adversas [Internet]. [cited 2016 Mar 17]. Available from: http://www. minsa.gob.pa/informacion-salud/centro-nacionalde-farmacovigilancia

26. Peru. Ministerio de la Salud. Reporte de sospecha de reacciones adversas a medicamentos [Internet]. 2015 [cited 2015 Dec 30]. Available from: http://www.digemid.minsa.gob.pe/upload/uploaded/pdf/FORMATO_REPORTE_PROF.PDF

27. Uruguay. Ministerio de la Salud Publica. Notificación de Sospecha de Reacción Adversa [Internet]. [cited 2015 Dec 30]. Available from: http://www2.msp.gub. uy/uc_3831_1.html

28. Venezuela. Ministerio del Poder Popular para la Salud. Sistema de Reporte de Reacciones Adversas o Eventos Adversos a Medicamentos [Internet]. [cited $2015 \mathrm{Dec}$ 30]. Available from: http://www.inhrr.gob.ve/ram1. php

29. Agência Nacional de Vigilância Sanitária (Anvisa). Notivisa - Sistema de Notificação em Vigilância Sanitária [Internet]. Brasília: Anvisa; [cited 2015 Dec 2]. Available from: http://www.anvisa.gov.br/hotsite/notivisa/apresenta.htm

30. Agência Nacional de Vigilância Sanitária (Anvisa). Manual do Usuário Versão 7 NOTIVISA - Sistema Nacional de Notificações para a Vigilância Sanitária - Módulo de Notificação. Brasília: Anvisa; 2008.

31. Agência Nacional de Vigilância Sanitária (Anvisa). Farmacovigilância no Brasil. Brasilia: Anvisa; s/data.

32. Fox AJ, Boye T, Calza S, Chang A, Grosjean P, Heiberger R, Pour KK, Kerns GJ, Lancelot R, Lesnoff M, Ligges U, Messad S, Maechler M, Muenchen R, Murdoch D, Neuwirth E, Putler D, Ripley B, Ristic M, Wolf P, Wright K. Package "Rcmdr"; 2009. p. 1-44.

33. Husson AF, Josse J, Le S, Mazet J, Husson MF. Package “FactoMineR"; 2016. p. 1-96.

34. Ferner RE. Causality Assessment in an Evolving Pharmacovigilance Landscape. In: Organization WH, editor. Determining causality case by case. Uppsala: World Health Organization; 2012. p. 7.

35. Ehrenstein V, Sørensen HT, Bakketeig LS, Pedersen L. Medical databases in studies of drug teratogenicity: Methodological issues. Clin Epidemiol 2010; 2:37-43.

36. Graham DJ, Ahmad SR, Piazza-Hepp T. Spontaneous Reporting - United States. In: Mann RD, Andrews E, editors. Pharmacovigilance. $2^{\text {nd }}$ ed. Chichester: John Wiley \& Sons Ltd; 2007. p. 237-247.

37. Varricchio F, Iskander J, Destefano F, Ball R, Pless R, Braun MM, Chen RT. Understanding vaccine safety information from the Vaccine Adverse Event Reporting System. Pediatr Infect Dis J 2004; 23(4):287-294.

38. Getz KA, Stergiopoulos S, Kaitin KI. Evaluating the Completeness and Accuracy of MedWatch Data. Am $J$ Ther 2014; 21(6):442-446.

39. Said Q. Outras questões metodológicas. In: Yang Y, West-Strum D, editors. Compreendendo a farmacoepidemiologia. Porto Alegre: AMGH; 2013. p. 105-120.
40. Edwards IR. Considerations on causality in pharmacovigilance. Int J Risk Saf Med 2012; 24(1):41-54.

41. World Health Organization (WHO). Preliminary Version of Minimal Information Model for Patient Safety. Geneva: WHO; 2014.

42. Mota DM, Kuchenbecker RS. Causalidade em farmacoepidemiologia e farmacovigilância: uma incursão teórica. Rev Bras Epidemiol 2017; 20(3):475-486.

43. Centers for Disease Control and Prevention. Updated guidelines for evaluating public health surveillance systems: recommendations from the guidelines working group. MMWR 2001; 50(RR-13):1-35.

44. European Centre for Disease Prevention and Control (ECDPC). Data quality monitoring and surveillance system evaluation. Stockolm: ECDPC; 2014.

45. Bailey C, Peddie D, Wickham ME, Badke K, Small SS, Doyle-Waters MM, Balka E, Hohl CM. Adverse drug event reporting systems: a systematic review. $\mathrm{Br} \mathrm{J} \mathrm{Clin}$ Pharmacol 2016; 82(1):17-29.

46. Agência Nacional de Vigilância Sanitária (Anvisa). VigiMed: registre problemas com medicamentos. [Internet]. [cited 2018 Feb 23]. Available from: http:// portal.anvisa.gov.br/rss/-/asset_publisher/Zk4q6UQCj9Pn/content/id/5238428

Artigo apresentado em 18/12/2018

Aprovado em 05/06/2019

Versão final apresentada em 07/06/2019

Editores-chefes: Romeu Gomes, Antônio Augusto Moura da Silva 
